\title{
CADERNOS DO IME - Série Estatística
}

Universidade do Estado do Rio de Janeiro - UERJ

Rio de Janeiro - RJ - Brasil

ISSN 1413-9022 / v. 28 p. 15 - 31, 2010

\section{UM MODELO DE PREVISÃO DO CONSUMO RESIDENCIAL DE ENERGIA ELÉTRICA NO BRASIL}

\author{
Beatriz Helena Assis Mascarenhas de Oliveira \\ IBMEC - RJ \\ Centro de Pesquisas de Energia Elétrica (CEPEL) \\ beatrizhelenadeoliveira@gmail.com \\ Jorge Machado Damázio \\ Universidade do Estado do Rio de Janeiro (UERJ) \\ Centro de Pesquisas de Energia Elétrica (CEPEL) \\ damazio@cepel.br \\ Rodrigo José Guerra Leone \\ Universidade Potiguar (UnP) \\ r.leone@uol.com.br \\ Mihail Lermontov \\ Universidade Federal Fluminense (UFF) \\ mihail@lermontov.com \\ Maria Augusta Soares Machado \\ IBMEC - RJ \\ mmachado@ibmecrj.br
}

\begin{abstract}
Resumo
O objetivo deste trabalho foi o desenvolvimento de um modelo previsão do consumo residencial de energia elétrica no Brasil. Dentre os métodos de previsão de demanda existentes, a metodologia adotada neste trabalho baseou-se na abordagem econométrica. $O$ método aplicado consistiu na modelagem de um vetor autoregressivo e de um modelo de correção de erros vetoriais, utilizando-se os procedimentos de estimação e testes desenvolvidos por Johansen (1988 e 1991) e Johansen e Juselius (1990). Para o período analisado, as elasticidades preço e renda de longo prazo da demanda residencial de energia elétrica foram de -0,3912 e 0,9649 respectivamente.
\end{abstract}

Palavras-chave: Previsão de consumo; Energia elétrica; Estimação. 


\section{Introdução}

A compreensão da importância dos modelos de previsão de consumo de energia elétrica requer uma breve explicação sobre o funcionamento do Setor Elétrico Brasileiro.

Fortunato et al. (2001) classifica os sistemas elétricos em três grandes grupos: hidrelétricos, termoelétricos e hidrotérmicos. Em alguns países, o aproveitamento de recursos hidrelétricos de porte, aliado à possibilidade de compra de energia de sistemas vizinhos, resulta em sistemas exclusivamente hidrelétricos. Em outros países, as condições geográficas impossibilitam a existência de potencial hidrelétrico, originando sistemas exclusivamente termoelétricos. Na maioria dos países, contudo, coexistem os dois tipos de geração, que caracterizam os sistemas hidrotérmicos. Isto porque a geração hidrelétrica está sempre exposta à possibilidade de estiagens recorrentes, quando a capacidade de geração fica sensivelmente diminuída, sendo então necessário recorrer à construção de grandes reservatórios que armazenam água durante os períodos de grandes chuvas para uso durante as estiagens e/ou ao acoplamento de usinas térmicas que são utilizadas quando a água armazenada pelo sistema hidrelétrico cai a níveis muito baixos.

O sistema brasileiro de produção de energia elétrica é um sistema hidrotérmico, com forte predominância de geração hidrelétrica e grande capacidade de armazenamento de água. $\mathrm{O}$ imenso potencial hidrelétrico brasileiro enfatiza a necessidade do planejamento da expansão da geração, tendo em vista que os prazos de construção de usinas hidroelétricas são bem maiores que os de usinas termoelétricas. $\mathrm{O}$ planejamento da operação tem também maior importância, dada a necessidade de coordenação das produções hidro e térmicas no controle do esvaziamento dos reservatórios. Neste contexto, é necessário avaliar com antecedência as condições de atendimento da demanda pelo sistema gerador. Por isso, no planejamento da expansão e no planejamento da operação trabalha-se com modelos de previsão de consumo de energia elétrica.

O objetivo principal ${ }^{1}$ deste trabalho é o desenvolvimento de um modelo de previsão do consumo residencial de energia elétrica no Brasil. Elasticidades preço e

\footnotetext{
${ }^{1}$ Maiores detalhes podem ser encontrados em De Oliveira (2004).
} 
renda são também estimadas. Estas elasticidades indicam como o consumo residencial de energia elétrica reage às variações de preço e renda.

\section{Revisão da Literatura}

\subsection{Modelos Top-Down e Bottom-Up}

Antes da primeira crise de petróleo, os modelos de planejamento energético se limitavam à determinação da oferta de energia. A demanda de energia era tratada como uma variável exógena e a oferta de energia era determinada por modelos de otimização (COSTA \& FALLOT, 2002).

As mudanças ocorridas após a crise de petróleo de 1973-1974 provocaram variações importantes na elasticidade-renda do consumo de energia, cujo comportamento anterior era praticamente constante. Dentre estas mudanças, cabe destacar:

- crescimento do setor de serviços nos países desenvolvidos e transferência de indústrias intensivas em energia para os países em desenvolvimento;

- substituição de fontes energéticas;

- inovações tecnológicas.

Estas mudanças levaram ao aprimoramento dos modelos existentes e ao desenvolvimento de novos modelos. A partir de então, o debate entre os especialistas em energia concentrou-se em duas correntes de modelos. De um lado, os modelos TopDown (TD). Do outro lado, os modelos Bottom-Up (BU).

A forma de representação do progresso técnico parece ser a principal diferença metodológica entre estas duas correntes de modelos (GRUBB et al., 1993, apud COSTA \& FALLOT, 2002). Os modelos TD representam o progresso técnico de uma forma bastante agregada, enquanto que os modelos $\mathrm{BU}$ fazem uma representação altamente desagregada, com uma especificação tecnológica detalhada.

Atualmente, verifica-se uma tendência no sentido de integrar estas duas correntes dentro de um mesmo modelo. O desenvolvimento de modelos denominados de híbridos tem sido possível, em consequência da identificação dos pontos fortes e fracos de cada corrente, além da não incompatibilidade entre as estruturas dos modelos (COSTA \& AMERICANO, 1996). 


\subsection{Principais Artigos Nacionais}

Modiano (1984) analisou a evolução histórica do consumo e da tarifa média de energia elétrica das classes residencial, industrial, comercial e outros no período 1963/1981. Com o propósito de avaliar quantitativamente a resposta da demanda de energia elétrica à variações da renda real e da tarifa real para as quatro classes distintas de consumidores, foram especificados e testados econometricamente dois modelos convencionais de determinação da demanda, ambos pressupondo que a oferta é infinitamente elástica. O primeiro modelo supôs que o ajustamento do consumo à demanda fosse instantâneo. O segundo modelo admitiu o ajustamento parcial do consumo à demanda, tendo sido estimadas as elasticidades renda e elasticidades preço de curto e longo prazos. As equações foram estimadas pelo método de mínimos quadrados com correção para correlação serial pelo método de Cochrane-Orcutt.

Araújo (1988) discutiu as relações entre modelos e o processo de planejamento, sistematizando as etapas do processo com ênfase na utilização de cenários e modelos nesse processo. Inicialmente, discutiu as ligações entre energia e sociedade, energia e desenvolvimento e introduziu conceitos básicos para o estudo de modelos como instrumentos de análise da realidade. Na segunda parte do trabalho, fez uma tipologia e análise dos principais enfoques, segundo finalidades, mantendo a divisão familiar entre modelos de demanda, oferta e integrados. Dentro desta divisão, distinguiu os diversos instrumentais utilizados, bem como a abrangência e outros aspectos relevantes. O ponto focal desta parte foram as hipóteses subjacentes e implicações dos modelos, buscando explicitar os conceitos envolvidos. Na terceira parte apresentou os métodos clássicos de previsão, seus pressupostos e aplicabilidade. Discutiu métodos de prospectiva também conhecidos como métodos de cenários. Em seguida, apresentou alguns dos principais procedimentos para construção de cenários, analisando a utilização do método como suporte de planejamento, introduzindo a questão do risco. No último capítulo concentrou-se no processo de planejamento propriamente dito, focando no uso de modelos: seu papel, limitações, a escolha de métodos adequados ao problema, integração entre análise e implementação, execução do estudo e a crítica dos mesmos e extração de conclusões válidas para elaboração de um plano. No final, retomou a discussão inicial, dando atenção a aspectos institucionais e à ligação entre a natureza do planejamento energético e a existência de um projeto global de desenvolvimento. 
Araújo (1992) realizou estudo econométrico da demanda de energia elétrica no Brasil para os setores industrial, residencial e outros consumos. A hipótese subjacente do estudo foi de que a demanda de eletricidade é mediada pelo estoque de equipamentos. No trabalho considera-se que, para cada categoria de usuários, variáveis renda e preços atuam sobre os estoques de equipamentos (através de compras de novos equipamentos e sucateamento dos velhos) e sobre o nível de sua utilização, a forma de atuação dependendo da categoria. No estudo foram feitas regressões nos logaritmos, obtendo-se elasticidades preço e elasticidades renda e foram testadas suas significâncias e a presença de autocorrelação serial nos resíduos. Sendo esta correlação significativa, prosseguiu com uma estimação em dois estágios. No caso do setor residencial, fez também a estimação de um modelo de defasagem distribuída. Vale mencionar que estas duas estimações correspondem a duas hipóteses distintas de como a inércia manifestase: a estimação em dois estágios supõe que a inércia aparece nos desvios entre a regressão (i.e., o valor esperado do consumo, dados preços e renda) e o valor observado; já a estimação por defasagem distribuída (modelo de ajuste parcial) implica em que o nível do consumo atual depende do consumo passado.

Andrade e Lobão (1997) analisaram a evolução do consumo residencial de energia elétrica no Brasil no período 1963/1965 e estimaram as elasticidades renda e elasticidades preço de sua demanda agregada. Além de atualizar parte do estudo feito por Modiano (1984), foram aplicados métodos econométricos mais eficientes para analisar a sensibilidade da função demanda residencial deste serviço de utilidade pública. A equação para demanda residencial de energia elétrica foi estimada por três diferentes métodos. O primeiro a ser utilizado foi o método de mínimos quadrados ordinários (MQO) sob a hipótese do modelo linear geral. Entretanto, os autores argumentam que como se trata da estimação de um modelo de demanda, é provável que a hipótese de correlação nula entre regressor e erro, fortemente requerida neste contexto de estimação, poderia estar sendo violada devido à existência de uma eventual simultaneidade entre o consumo e a tarifa de energia elétrica. Por este motivo, apresentam uma estimação de variáveis instrumentais (VI), do tipo dois estágios, com o intuito de corrigir os possíveis vieses gerados pela estimação direta de MQO. O terceiro método aplicado consistiu na modelagem de um vetor autoregressivo sob a representação de um modelo de correção de erros, utilizando-se os procedimentos de 
estimação e testes desenvolvidos por Johansen (1988 e 1991) e ainda Johansen e Juselius (1990).

Schmidt e Lima (2002) estimaram as elasticidades, principalmente as preço e renda, de longo prazo da demanda por energia elétrica nas três classes de consumo: residencial, comercial e industrial. Os autores apresentam um modelo microeconômico a ser utilizado nas estimações e uma resenha referente a estudos sobre elasticidades a nível internacional e nacional. O método de estimação utilizado no trabalho também foi o de Johansen (1988 e 1991) e Johansen e Juselius (1990); isto é, o de cointegração, que requer a modelagem de um vetor autoregressivo, sob a representação de um modelo de correção de erro vetorial, para levar em consideração a não estacionariedade das variáveis do modelo.

\section{Metodologia}

\subsection{Tipo de Abordagem}

Dentre os principais métodos de previsão de demanda existentes, a metodologia adotada neste trabalho baseou-se na abordagem econométrica. Convém lembrar que esta abordagem busca analisar a evolução temporal de uma variável dependente ou explicada, em função da evolução temporal de variáveis independentes ou explicativas.

Mais especificamente, o modelo de previsão de demanda desenvolvido visa quantificar as relações existentes entre o consumo residencial de energia elétrica e o Produto Interno Bruto (PIB), a tarifa real média residencial de energia elétrica e o preço de eletrodomésticos.

A estimação dos parâmetros foi feita através de método estatístico, utilizando-se as séries históricas anuais das variáveis do modelo. O método aplicado consistiu na modelagem de um vetor autoregressivo e de um modelo de correção de erros vetoriais, utilizando-se os procedimentos de estimação e testes desenvolvidos por Johansen (1988 e 1991) e Johansen e Juselius (1990).

\subsection{Dados e Fontes Utilizados}

A Tabela 1 mostra os dados do consumo residencial de energia elétrica, do PIB, da tarifa média residencial de energia elétrica e do preço de eletrodomésticos no período 1980-2003. 
Tabela 1 - Variáveis do Modelo

\begin{tabular}{|c|c|c|c|c|}
\hline Ano & $\begin{array}{c}\text { Consumo } \\
\text { Residencial }\end{array}$ & PIB & $\begin{array}{c}\text { Tarifa Média } \\
\text { Residencia }\end{array}$ & $\begin{array}{c}\text { Preços de } \\
\text { Eletrodomésticos }\end{array}$ \\
\hline 1980 & GWh & R $\$$ Milhões de 2003 & /MW & 10,31 \\
1981 & 23.184 & 968371,28 & 330,37 & 11,35 \\
1982 & 27.960 & 927215,50 & 315,20 & 11,76 \\
1983 & 29.742 & 934911,39 & 293,33 & 9,80 \\
1984 & 30.923 & 956524,48 & 258,23 & 8,45 \\
1985 & 32.624 & 1031602,09 & 233,42 & 8,51 \\
1986 & 35.747 & 1108869,09 & 217,64 & 7,89 \\
1987 & 38.343 & 1148012,17 & 188,53 & 6,65 \\
1988 & 40.536 & 1147323,36 & 229,40 & 8,87 \\
1989 & 43.655 & 1183578,78 & 212,61 & 5,07 \\
1990 & 47.951 & 1132093,10 & 160,41 & 3,74 \\
1991 & 51.108 & 1143753,66 & 206,10 & 3,35 \\
1992 & 51.901 & 1137536,44 & 215,42 & 2,82 \\
1993 & 53.626 & 1193557,45 & 238,13 & 2,24 \\
1994 & 55.954 & 1263414,82 & 221,17 & 1,95 \\
1995 & 63.576 & 1316778,86 & 201,83 & 1,72 \\
1996 & 69.051 & 1351786,61 & 185,03 & 1,52 \\
1997 & 74.089 & 1396008,80 & 232,88 & 1,38 \\
1998 & 79.339 & 1397850,36 & 242,46 & 1,32 \\
1999 & 81.293 & 1408830,11 & 245,82 & 1,20 \\
2000 & 83.614 & 1470264,51 & 243,14 & 1,10 \\
2001 & 73.622 & 1489562,96 & 244,38 & 1,08 \\
2002 & 72.661 & 1518264,12 & 250,58 & 1,00 \\
2003 & 76.168 & 1514923,94 & 239,30 & \\
\hline
\end{tabular}

A fonte dos dados do consumo residencial foi o Banco Central (BC). A tarifa média residencial de energia elétrica foi deflacionada pelo IGP-DI. A fonte dos dados da tarifa média residencial foi o Instituto de Pesquisa Econômica Aplicada (IPEA) para o período 1980-1994 e ANEEL (1995-2003). A série do IGP-DI foi coletada no IPEA. A série do PIB a preços constantes de 2003 foi coletada no BC. A série dos preços de eletrodomésticos foi obtida deflacionando o IPA-OG-eletrodomésticos pelo IGP-DI. A fonte dos dados foi o IPEA.

O Quadro 1 sintetiza os indicadores utilizados na modelagem e as fontes dos dados.

Quadro 1 - Fontes dos Dados

\begin{tabular}{|l|l|}
\hline \multicolumn{1}{|c|}{ Indicadores } & Fontes \\
\hline Consumo Residencial de Energia Elétrica & BC \\
\hline IGP-DI & IPEA \\
\hline IPA-OG-eletrodomésticos & IPEA \\
\hline PIB & BC \\
\hline Tarifa Média Residencial de Energia Elétrica & IPEA \\
\hline
\end{tabular}


Após transformar os dados brutos para índice em base fixa (1980-2003), o Gráfico 1 foi plotado para a visualização da evolução das variáveis do modelo.

\section{Gráfico 1 - Evolução das Variáveis do Modelo}

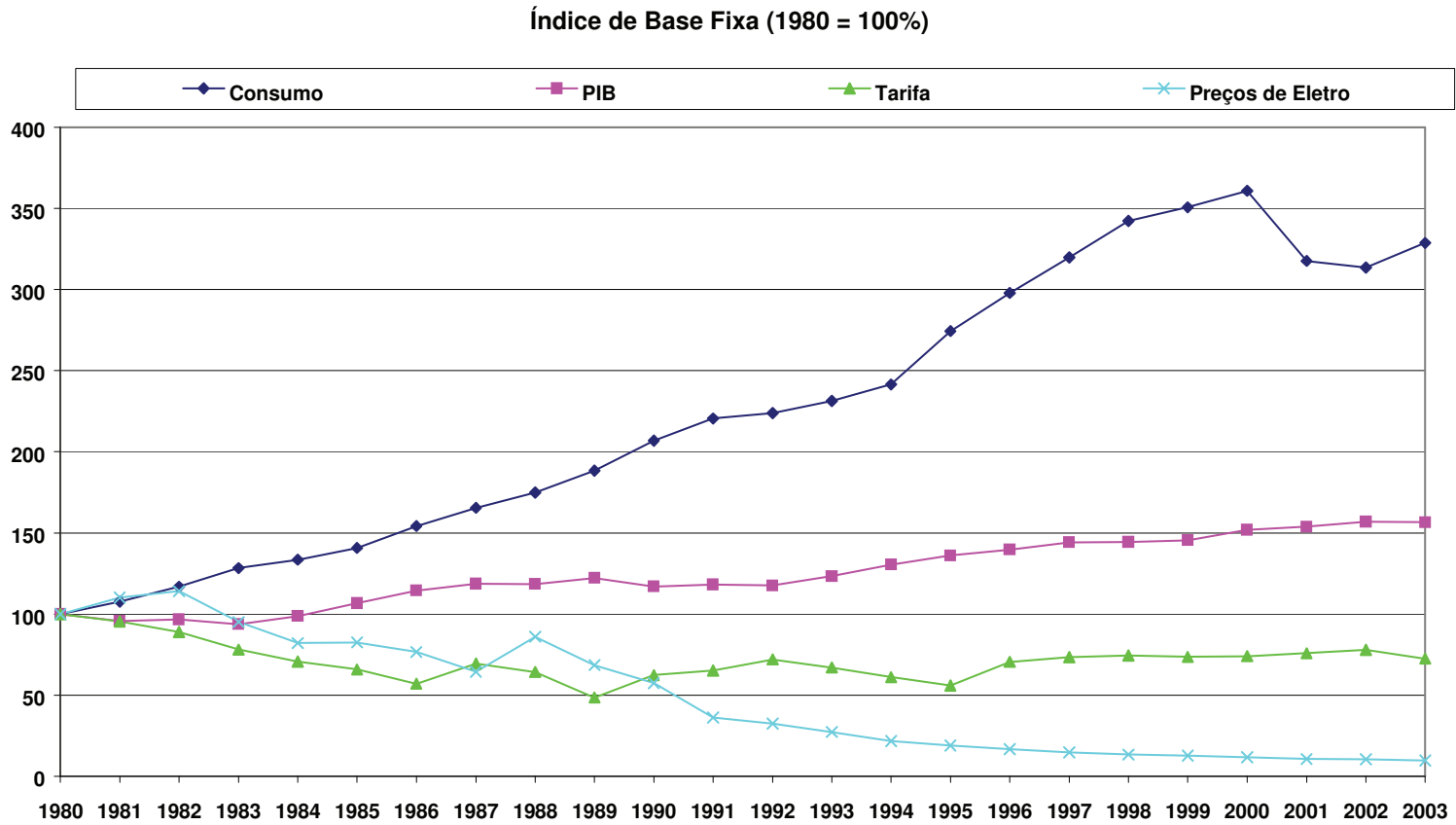

\section{Testes e Estimação do Modelo}

O modelo teórico foi construído utilizando-se a seguinte premissa: Toda quantidade de energia elétrica demandada pelos consumidores residenciais é efetivamente fornecida. Dito de outra maneira, de uma forma geral ou para grande parte dos consumidores, admite-se que a oferta do serviço seja infinitamente elástica. Com esta premissa, pode-se utilizar a quantidade consumida com uma boa aproximação para a quantidade demandada (ANDRADE \& LOBÃO, 1997).

Cabe destacar que, dada a premissa de oferta infinitamente elástica, na estimação do modelo não foram considerados os dados do período 2001-2003, pois houve racionamento de energia elétrica (restrição de oferta) nos anos de 2001 e 2002.

A equação de demanda residencial de energia elétrica foi especificada da seguinte forma funcional (SCHMIDT \& LIMA, 2002):

$\mathrm{C}_{\mathrm{t}}=\mathrm{K} \cdot \mathrm{P}_{\mathrm{t}}^{\alpha} \cdot \mathrm{Y}_{\mathrm{t}}^{\beta} \cdot \mathrm{PE}_{\mathrm{t}}^{\delta}$

em que: $K>0, \alpha<0, \beta>0$ e $\delta<0$ 
onde:

$\mathrm{C}_{\mathrm{t}}$ : consumo residencial de energia elétrica no tempo $\mathrm{t}$;

$\mathrm{P}_{\mathrm{t}}: \quad$ tarifa residencial de energia elétrica no tempo $\mathrm{t}$;

$\mathrm{Y}_{\mathrm{t}}: \quad$ renda no tempo t;

$\mathrm{PE}_{\mathrm{t}}$ : preço de eletrodomésticos no tempo t;

Tomando logaritmo na equação (4.1), chega-se à seguinte equação linear para a demanda residencial de energia elétrica:

$\log C_{t}=\log \mathrm{K}+\alpha \log \mathrm{P}_{t}+\beta \log \mathrm{Y}_{\mathrm{t}}+\delta \log \mathrm{PE}_{\mathrm{t}}$

em que: $\mathrm{K}>0, \alpha<0, \beta>0$ e $\delta<0$

A escolha da forma funcional log-linear deveu-se ao fato de que as elasticidades são obtidas diretamente dos resultados da regressão e, diferentemente das formas lineares, são constantes e independentes dos valores assumidos pelas variáveis. Doravante, $\quad \mathrm{C}_{\mathrm{t}}, \mathrm{P}_{\mathrm{t}}, \mathrm{Y}_{\mathrm{t}}$ e $\mathrm{PE}_{\mathrm{t}} \quad$ representam, respectivamente, $\log C_{t}, \log P_{t}, \log Y_{t}$ e $\log P E_{t}$

Teoricamente, espera-se que o consumo reaja negativamente aos aumentos da tarifa residencial e do preço de eletrodomésticos e, positivamente, aos aumentos de renda. Os coeficientes $\alpha, \beta$ e $\delta$ representam, respectivamente, as elasticidades da demanda residencial de energia elétrica com relação ao preço de energia elétrica, renda e preço de eletrodomésticos.

$\mathrm{Na}$ estimação foram utilizados dados de séries temporais. Uma hipótese implícita na análise de regressão envolvendo dados de séries temporais é que tais dados são estacionários.

\subsection{Teste de Raiz Unitária}

O teste de raiz unitária é um teste para detectar não-estacionariedade. Neste trabalho, o teste foi realizado utilizando-se o software Eviews.

De acordo com Gujarati (2000), tem-se o seguinte modelo:

$\mathrm{Y}_{\mathrm{t}}=\mathrm{Y}_{\mathrm{t}-1}+\mathrm{u}_{\mathrm{t}}$

onde:

$\mathrm{u}_{\mathrm{t}}$ é o termo de erro estocástico que tem média zero, variância $\sigma^{2}$ constante e é nãoautocorrelacionado. 
Conforme ainda Gujarati (2000), a equação (4.3) é similar a uma regressão de primeira ordem, ou $\mathrm{AR}(1)$, onde o valor $Y$ no instante $t$ é regredido sobre seu valor no instante $(t-1)$. Se o coeficiente da regressão de $Y_{t-1}$ for igual a 1 , tem-se o problema da raiz unitária; ou seja, uma situação de não estacionariedade. A variável estocástica $Y$ tem uma raiz unitária, se ao se aplicar a regressão

$$
\mathrm{Y}_{\mathrm{t}}=\rho \mathrm{Y}_{\mathrm{t}-1}+\mathrm{u}_{\mathrm{t}}
$$

obtém-se $\rho=1$.

A equação (4.4) pode ser reescrita como

$$
\Delta Y_{t}=(\rho-1) Y_{t-1}+u_{t}=\delta Y_{t-1}+u_{t}
$$

onde:

$\delta=(\rho-1)$ e $\Delta$ é o operador de primeira diferença.

Se $\delta$ for 0 , obtém-se:

$$
\Delta \mathrm{Y}_{\mathrm{t}}=\left(\mathrm{Y}_{\mathrm{t}}-\mathrm{Y}_{\mathrm{t}-1}\right)=\mathrm{u}_{\mathrm{t}}
$$

Ou seja, as primeiras diferenças da série temporal $Y_{t}$ é uma série temporal estacionária.

Convenciona-se chamar uma série temporal integrada de ordem $d$, ou $\mathrm{I}(d)$, se for necessário diferenciá-la $d$ vezes para torná-la estacionária. Uma série é não estacionária se for integrada de ordem maior ou igual a 1.

Pode-se verificar a estacionariedade de uma série temporal $Y_{t}$, aplicando (4.4) e testando a hipótese nula $\hat{\rho}$ igual a 1 com base na estatística $t$. Neste caso, $t$ é conhecida por estatística $\tau$ (tau) e seus valores críticos foram tabulados por Dickey e Fuller, utilizando-se simulações de Monte Carlo. O teste tau é conhecido como teste de Dickey-Fuller (DF).

O teste de Dickey-Fuller é feito verificando-se a hipótese nula $\delta=0$ nas seguintes regressões:

$$
\begin{aligned}
& \Delta \mathrm{Y}_{\mathrm{t}}=\delta \mathrm{Y}_{\mathrm{t}-1}+\mathrm{u}_{\mathrm{t}} \\
& \Delta \mathrm{Y}_{\mathrm{t}}=\beta_{1}+\delta \mathrm{Y}_{\mathrm{t}-1}+\mathrm{u}_{\mathrm{t}} \\
& \Delta \mathrm{Y}_{\mathrm{t}}=\beta_{1}+\beta_{2} \mathrm{t}+\delta \mathrm{Y}_{\mathrm{t}-1}+\mathrm{u}_{\mathrm{t}}
\end{aligned}
$$

onde:

$t$ é a variável tempo ou tendência. 
Se o termo de erro $u_{t}$ é autocorrelacionado, utiliza-se termos de diferenças defasados - teste aumentado de Dickey-Fuller (ADF).

$$
\Delta \mathrm{Y}_{\mathrm{t}}=\beta_{1}+\beta_{2} \mathrm{t}+\delta \mathrm{Y}_{\mathrm{t}-1}+\alpha_{\mathrm{i}} \sum_{\mathrm{i}=1}^{\mathrm{m}} \Delta \mathrm{Y}_{\mathrm{t}-1}+\varepsilon_{\mathrm{t}}
$$

Com o objetivo de testar a ordem de integração das séries temporais das variáveis do modelo, foi realizado o teste ADF. Os resultados do teste foram os seguintes:

Tabela 2 - Teste ADF nas Séries Temporais

\begin{tabular}{|c|c|c|c|c|c|}
\hline Variável & Equação de Teste & Defasagem & $\begin{array}{c}\text { Estatística } \\
\text { de Teste }\end{array}$ & \multicolumn{2}{|c|}{$\begin{array}{c}\text { Valor Crítico } \\
\text { (MacKinnon) }\end{array}$} \\
\cline { 4 - 6 } & & & & $\mathbf{1 \%}$ & $\mathbf{5 \%}$ \\
\hline $\mathrm{C}_{\mathrm{t}}$ & constante & 1 & $-1,0885$ & $-3,8315$ & $-3,0300$ \\
$\mathrm{P}_{\mathrm{t}}$ & sem constante e sem tendência & 0 & $-0,5848$ & $-2,6857$ & $-1,9591$ \\
$\mathrm{Y}_{\mathrm{t}}$ & sem constante e sem tendência & 1 & 2,2676 & $-2,6924$ & $-1,9602$ \\
$\mathrm{PE}_{\mathrm{t}}$ & constante & 0 & 0,4443 & $-3,8085$ & $-3,0207$ \\
\hline
\end{tabular}

Estes resultados indicam que a hipótese nula de que existe uma raiz unitária não pode ser rejeitada ao nível de significância de 5\%; ou seja, todas as séries temporais podem ser consideradas não-estacionárias.

Em seguida, foram realizados testes de raiz unitária nas primeiras diferenças (Tabela 3), tendo sido constatado que as séries diferenciadas uma vez podem ser estacionárias ao nível de significância de 5\%. Foi concluído que todas as séries temporais podem ser consideradas como integradas de ordem 1; ou seja, I(1).

Tabela 3 - Teste ADF nas Séries Temporais em Primeira Diferença

\begin{tabular}{|c|c|c|c|c|c|}
\hline Variável & Equação de Teste & Defasagem & $\begin{array}{c}\text { Estatística de } \\
\text { Teste }\end{array}$ & \multicolumn{2}{|c|}{$\begin{array}{c}\text { Valor Crítico } \\
\text { (MacKinnon) }\end{array}$} \\
\cline { 4 - 6 } & & & & $\mathbf{1 \%}$ & $\mathbf{5 \%}$ \\
\hline $\mathrm{C}_{\mathrm{t}}$ & constante & 1 & $-3,3539$ & $-3,8574$ & $-3,0404$ \\
\hline $\mathrm{P}_{\mathrm{t}}$ & sem constante e sem tendência & 0 & $-4,7617$ & $-2,6924$ & $-1,9602$ \\
\hline $\mathrm{Y}_{\mathrm{t}}$ & sem constante e sem tendência & 0 & $-2,4480$ & $-2,6924$ & $-1,9602$ \\
\hline $\mathrm{PE}_{\mathrm{t}}$ & constante & 0 & $-4,7317$ & $-3,8315$ & $-3,0300$ \\
\hline
\end{tabular}




\subsection{Teste de Co-Integração}

Conforme mencionado anteriormente, uma hipótese implícita na análise de regressão envolvendo dados de séries temporais é que tais dados são estacionários. Uma vez constatado que as séries temporais das variáveis do modelo são não-estacionárias, o método estatístico utilizado neste trabalho consistiu na modelagem de um vetor autoregressivo e de um modelo de correção de erros vetoriais, utilizando-se os procedimentos de estimação e testes desenvolvidos por Johansen (1988 e 1991) e Johansen e Juselius (1990). No caso de modelagem de séries temporais nãoestacionárias, este método é o que fornece o tratamento estatístico mais adequado para estimar a função de demanda de longo prazo e para realizar previsões do consumo de energia elétrica (ANDRADE \& LOBÃO, 1997).

Engle e Granger (1987) apontaram que uma combinação linear de duas ou mais séries não-estacionárias pode ser estacionária. Se tal combinação linear existir, as séries não estacionárias são co-integradas. A combinação linear estacionária é chamada de equação de co-integração e pode ser interpretada como uma relação de equilíbrio de longo prazo entre as variáveis.

Dado que todas as séries temporais das variáveis do modelo têm a mesma ordem de integração, foi realizado o teste de co-integração para testar a existência de vetores de co-integração. Para a realização do teste de co-integração, é necessário que se defina a ordem do modelo VAR que será especificado. Utilizando o critério de Schwarz, concluiu-se que o modelo VAR deve ser especificado com apenas 1 defasagem das variáveis. Em seguida, utilizando o software EViews, foram realizados o teste $\lambda_{\text {traço }}$ e o teste $\lambda_{\text {max }}$. $\mathrm{O}$ teste $\lambda_{\text {traço }}$ indicou a existência de 2 vetores de co-integração ao nível de significância de 5\% e, apenas 1 vetor de co-integração ao nível de significância de $1 \%$.

Tabela $4-$ Teste $\lambda_{\text {traço }}$

\begin{tabular}{|c|c|c|c|c|}
\hline $\begin{array}{c}\mathbf{N}^{\mathbf{0}} \text { Vetores de } \\
\text { Co-integração } \\
(\mathbf{r})\end{array}$ & Eigenvalue & Estatística de Teste & \multicolumn{2}{|c|}{ Valor Crítico } \\
\cline { 3 - 5 } & & & $\mathbf{1 \%}$ & $\mathbf{5 \%}$ \\
\hline $\mathrm{r}=0$ & 0,9560 & 90,31 & 45,58 & 39,89 \\
\hline $\mathrm{r} \leq 1$ & 0,5812 & 27,85 & 29,75 & 24,31 \\
\hline $\mathrm{r} \leq 2$ & 0,4039 & 10,44 & 16,31 & 12,53 \\
\hline $\mathrm{r} \leq 3$ & 0,0047 & 0,09 & 6,51 & 3,84 \\
\hline
\end{tabular}


$\mathrm{O}$ teste $\lambda_{\text {max }}$, por sua vez, indicou a existência de apenas 1 vetor de cointegração aos nivéis de significância de $1 \%$ e $5 \%$.

Tabela 5 - Teste $\lambda_{\max }$

\begin{tabular}{|c|c|c|c|c|}
\hline $\begin{array}{c}\mathbf{N}^{\mathbf{0}} \text { Vetores de } \\
\text { Co-integração } \\
(\mathbf{r})\end{array}$ & Eigenvalue & Estatística de Teste & \multicolumn{2}{|c|}{ Valor Crítico } \\
\cline { 4 - 5 } & & & $\mathbf{1 \%}$ & $\mathbf{5 \%}$ \\
\hline $\mathrm{r}=0$ & 0,9560 & 62,46 & 28,82 & 23,80 \\
\hline $\mathrm{r} \leq 1$ & 0,5812 & 17,41 & 22,99 & 17,89 \\
\hline $\mathrm{r} \leq 2$ & 0,4039 & 10,35 & 15,69 & 11,44 \\
\hline $\mathrm{r} \leq 3$ & 0,0047 & 0,09 & 6,51 & 3,84 \\
\hline
\end{tabular}

Os dois testes de co-integração indicam que as variáveis são co-integráveis, identificando a existência de um vetor de co-integração ao nível de significância de $1 \%$. A relação de co-integração é dada pelo seguinte vetor normalizado:

Tabela 6 - Vetor Normalizado

\begin{tabular}{|c|c|c|c|}
\hline $\mathrm{C}_{\mathrm{t}}$ & $\mathrm{P}_{\mathrm{t}}$ & $\mathrm{Y}_{\mathrm{t}}$ & $\mathrm{PE}_{\mathrm{t}}$ \\
\hline 1,000 & 0,3912 & $-0,9649$ & 0,2784 \\
\hline
\end{tabular}

Então, a relação de longo prazo entre as variáveis do modelo fica estimada pela seguinte equação de co-integração:

$\mathrm{C}_{\mathrm{t}}=-0,3912 \mathrm{P}_{\mathrm{t}}+0,9649 \mathrm{Y}_{\mathrm{t}}-0,2784 \mathrm{PE}_{\mathrm{t}}$

A equação (4.10) indica que a elasticidade-preço de longo prazo da demanda residencial de energia elétrica é $-0,3912$, o que significa que um aumento de $1 \%$ da tarifa residencial de energia elétrica causará uma redução de $0,3912 \%$ no consumo residencial de energia elétrica. Da mesma forma, os coeficientes 0,9649 e -0,2784 representam, respectivamente, a elasticidade-renda de longo prazo da demanda residencial de energia elétrica e a elasticidade preço de eletrodomésticos de longo prazo da demanda residencial de energia elétrica.

\subsection{Modelo de Correção de Erros Vetoriais}

Com a finalidade de realizar previsões, foi estimado o modelo de correção de erros vetorias (MCEV). O MCEV é um VAR restrito utilizado em séries nãoestacionárias co-integráveis. O MCEV possui uma relação de co-integração em sua especificação, que restringe o comportamento de longo prazo das variáveis, permitindo ajustes na dinâmica de curto prazo. O termo de co-integração é conhecido como erro de 
correção, na medida em que os desvios do equilíbrio de longo prazo são corrigidos gradualmente através de uma série de ajustes parciais de curto prazo.

A equação de co-integração (4.10) foi utilizada para derivar o erro de correção defasado:

$$
\mathrm{u}_{\mathrm{t}-1}=\mathrm{C}_{\mathrm{t}-1}+0,3912 \mathrm{P}_{\mathrm{t}-1}-0,9649 \mathrm{Y}_{\mathrm{t}-1}+0,2784 \mathrm{PE}_{\mathrm{t}-1}
$$

Em seguida, foram testadas diversas especificações para $\Delta C_{t}$, acrescentando uma constante e as variáveis $\Delta \mathrm{P}_{\mathrm{t}}, \Delta \mathrm{Y}_{\mathrm{t}}, \Delta \mathrm{PE}_{\mathrm{t}}$ e $\Delta \mathrm{C}_{\mathrm{t}-1}$. Como a variável de interesse é o consumo residencial de energia elétrica, só foi estimado um MCEV para esta variável. O melhor resultado obtido foi o seguinte:

Tabela 7 - Estimação do MCEV

\begin{tabular}{|c|c|c|c|c|}
\hline Variável & Coeficiente & Desvio Padrão & Estatística t & Prob. \\
\hline Constante & $-0,0516$ & 0,0192 & $-2,6915$ & 0,0167 \\
\hline $\mathrm{u}_{\mathrm{t}-1}$ & $-0,3348$ & 0,0575 & $-5,8268$ & 0,0000 \\
\hline$\Delta \mathrm{C}_{\mathrm{t}-1}$ & 0,4157 & 0,1460 & 2,8467 & 0,0122 \\
\hline$\Delta \mathrm{PE}_{\mathrm{t}}$ & $-0,0615$ & 0,0290 & $-2,1189$ & 0,0512 \\
\hline \multicolumn{5}{|c|}{$\mathrm{R}^{2}=0,7264$} \\
0,7264 \\
F teste $=13,28(0,0002)$ \\
LM teste $=3,3208(0,1901)$ \\
Jarque-Bera teste $=0,6131(0,7360)$ \\
White teste $=3,6173(0,7283)$ \\
\hline \multicolumn{4}{|c|}{ Nota: Os valores entre parênteses representam o p-value. } \\
\hline
\end{tabular}

Os coeficientes são significativos e não existem indícios de que os resíduos não sejam normalmente distribuídos (Jarque-Bera teste), homocedásticos (White teste) e não-autocorrelacionados (LM teste).

Cabe ressaltar que a primeira diferença da variável preço $\left(\Delta \mathrm{P}_{\mathrm{t}}\right)$ e a primeira diferença da variável renda $\left(\Delta \mathrm{Y}_{\mathrm{t}}\right)$ não se mostraram significativas e por isso não fazem parte da equação do MCEV.

Desta maneira, o modelo pode ser estimado com a equação (4.12), que representa as dinâmicas de curto e longo prazos para o consumo residencial de energia elétrica:

$\Delta \mathrm{C}_{\mathrm{t}}=-0,0516-0,3348 \mathrm{u}_{\mathrm{t}-1}+0,4157 \Delta \mathrm{C}_{\mathrm{t}-1}-0,0615 \Delta \mathrm{PE}_{\mathrm{t}}$ 
O MCEV indica que a velocidade de ajustamento em relação ao equilíbrio de longo prazo é -0,3348; ou seja, 33,5\% do desequilíbrio de curto prazo em relação à trajetória de longo prazo são corrigidos a cada período. Isto significa que o ajuste total dos desvios do equilíbrio de longo prazo levaria cerca de 3 anos.

\section{Considerações Finais}

Este trabalho objetivou o desenvolvimento de um modelo de previsão do consumo residencial de energia elétrica no Brasil, importante ferramenta para o planejamento da expansão e o planejamento da operação do Setor Elétrico Brasileiro. Além disso, buscou contribuir para estimações de elasticidades preço e renda de longo prazo da demanda residencial de energia elétrica.

O método aplicado consistiu na modelagem de um vetor autoregressivo (VAR) sob a representação de um modelo de correção de erros vetoriais, utilizando-se os procedimentos de estimação e testes desenvolvidos por Johansen (1988 e 1991) e ainda Johansen e Juselius (1990).

Esta metodologia foi utilizada por Andrade e Lobão (1997) e Schmidt e Lima (2002), que analisaram a evolução do consumo residencial de energia elétrica no Brasil e estimaram as elasticidades preço e renda da demanda residencial de energia elétrica para diferentes períodos.

Cabe destacar que neste trabalho foram utilizadas as séries temporais do consumo total de energia elétrica e do PIB, diferenciando-se do adotado nos dois trabalhos mencionados que utilizaram as séries temporais do consumo por consumidor de energia elétrica e o PIB per capita. Por isso, as elasticidades preço e renda da demanda residencial de energia elétrica estimadas neste trabalho não são comparáveis com as encontradas anteriormente.

Por ultimo, vale ressaltar ainda que, dado que a restrição de oferta provocada pelo racionamento de energia elétrica de 2001-2002 viola a hipótese básica do trabalho de que a oferta do serviço seja infinitamente elástica, a estimação do modelo foi realizada com base nas observações do período 1980-2000. Para este período, as elasticidades preço e renda de longo prazo da demanda residencial de energia elétrica foram de $-0,3912$ e 0,9649 respectivamente. 


\section{Referências}

ANDRADE, T. A.; LOBÃO, W. J. A. Elasticidade Renda e Preço da Demanda Residencial de Energia Elétrica no Brasil. In: Texto para Discussão n 489, IPEA, p. 1-20, 1997.

ARAÚJO, J. L. R. H. Modelos de Energia para Planejamento. Tese (Concurso de Professor Titular), COPPE/UFRJ, 1984.

ARAÚJO, J. L. R. H. Estudo Econométrico da Demanda de Energia Elétrica no Brasil, Relatório Final, IE/UFRJ, 1992.

COSTA, R. C.; FALLOT, A. Top-down Versus Bottom-Up: Coupling Both Modelling Approaches for a Prospective Study on Biofuels. Comprehensive Economic and Spatial Bio-Energy Modelling. 48 ed. Chania: CIHEAM (Centre International de Hautes Etudes Agronomiques Méditerranéenes), 2002.

COSTA, R. C.; AMERICANO, B. B. "Modelos Top-Down e Bottom-Up: Reflexões sobre o Planejamento Energético". In: Congresso Brasileiro de Energia, Rio de Janeiro, 1996.

DE OLIVEIRA, B. H. A. M. (2004), "Um Modelo de Previsão do Consumo Residencial de Energia Elétrica no Brasil”. Monografia de Final do Curso de MBA Executivo em Finanças, IBMEC/RJ.

ENGLE, R. F.; GRANGER, C. W. J. Co-integration and Error Correction: Representation, Estimation, and Testing. In: Econometrica, Vol. 55, No. 2, p. 251-276, 1987.

FORTUNATO L. A. M.; ARARIPE NETO, T. A.; AlBUQUERQUE, J. C. R.; PEREIRA, M. V. F. Introdução ao Planejamento da Expansão e Operação de Sistemas de Produção de Energia Elétrica 1a. ed. Niterói: Atlas, 2001.

GUJARATI, D. N. Econometria Básica, São Paulo: MAKRON Books, 2000.

JOHANSEN, S. Statistical Analysis og Cointegrating Vectors. In: Journal of Economic Dynamics and Control, Vol. 12., p. 231-254, 1988.

JOHANSEN, S. Estimation and Hypothesis Testing of Cointegration Vectors in Gaussian Vector Autoregressive Models. In: Econometrica, Vol. 59, No. 6, p. 1551-1580, 1991.

JOHANSEN, S.; JUSELIUS, K.. Maximum Likelihood Estimation and Inference on Cointegration With Applications to the Demand for Money. In: Oxford Bulletin of Economics and Statistics, Vol. 52, No. 2, p. 169-210, 1990.

MODIANO, E. M. Elasticidade Renda e Preços da Demanda de Energia Elétrica no Brasil. In: Texto para Discussão n⿳0 68, Departamento de Economia da PUC, p. 1-19, 1984.

SCHMIDT, C. A. J.; LIMA, M. A. Estimações e Previsões da Demanda por Energia Elétrica no Brasil. In: Documento de Trabalho $\mathbf{n}^{\mathbf{0}}$ 16, Ministério da Fazenda. Secretaria de Acompanhamento Econômico SEAE, p. 1-31, 2002. 


\title{
A FORECASTING MODEL FOR RESIDENTIAL ELECTRICAL ENERGY CONSUMPTION IN BRAZIL
}

\begin{abstract}
The objective of this work was to develop a model to predict the residential electrical energy consumption in Brazil. Among the methods of demand forecasting in existence, the methodology adopted was based on an econometric approach. The method applied was based on the modeling of an autoregressive vector and an error correction vector, using the estimation procedures and tests developed by Johansen (1988 and 1991) and Johansen and Juselius (1990). For the period analyzed, the price and income elasticities of demand for long-term residential electricity were -0.3912 and 0.9649 respectively.

Key-words: Forecast; Electrical energy; Estimation.
\end{abstract}

